\title{
P63 Accuracy (Validation) of Central Blood Pressure Measurement Using the Sphygmocor Xcel-cuff Device
}

\author{
Martin Schultz ${ }^{1, *}$, Dean Picone ${ }^{1}$, Matthew Armstrong ${ }^{1}$, Andrew Black ${ }^{2}$, Nathan Dwyer ${ }^{2}$, \\ Philip Roberts-Thomson ${ }^{2}$, James Sharman ${ }^{1}$
}

${ }^{1}$ Menzies Institute for Medical Research, University of Tasmania, Hobart, Australia

${ }^{2}$ Royal Hobart Hospital, Hobart, Australia

\section{ABSTRACT}

Background: Numerous devices purport to measure central aortic BP as distinct from conventional brachial BP. This study aimed to determine the accuracy (validation) of the Sphygmocor Xcel-cuff device (AtCor Medical, Sydney, Australia) for measuring central BP.

Methods: 330 patients (mean age $61.3 \pm 10.6$ years) undergoing coronary angiography had simultaneous measurement of invasive aortic BP and non-invasive cuff-derived central BP using the Xcel device (total $n=552$ individual comparisons). Methods were undertaken according to Artery Society guidelines and several calibration techniques to derive central SBP were examined.

Results: Central SBP was significantly underestimated, and with wide variability, when using the default calibration of brachial cuff SBP/DBP $(-7.7 \pm 11.0 \mathrm{mmHg}$ ). Similar wide variability was observed using other calibration methods (cuff $33 \%$ formfactor MAP/DBP, $-4.4 \pm 11.5 \mathrm{mmHg}$; cuff $40 \%$ form-factor MAP/DBP, $4.7 \pm 11.9 \mathrm{mmHg}$; cuff oscillometric MAP/DBP, $-18.2 \pm$ $12.1 \mathrm{mmHg})$. Only calibration with invasive aortic integrated MAP/DBP resulted in a mean difference \pm SD $(3.3 \pm 7.5 \mathrm{mmHg})$ within the minimum tolerable error of $\leq 5 \pm \leq 8 \mathrm{mmHg}$. The difference between brachial cuff SBP and invasive aortic SBP was $3.3 \pm 10.7 \mathrm{mmHg}$. A subsample $(n=151)$ analysis to determine the accuracy of central-to-brachial SBP amplification, showed this to be over-estimated by the Xcel device $(4.3 \pm 9.1 \mathrm{mmHg}, p=0.02)$.

Conclusion: Irrespective of calibration technique, the Sphygmocor Xcel-cuff device does not pass the Artery Society accuracy (validation) criteria for non-invasive measurement of central BP. Further accuracy refinements of this device are required.

(c) 2019 Association for Research into Arterial Structure and Physiology. Publishing services by Atlantis Press International B.V. This is an open access article distributed under the CC BY-NC 4.0 license (http://creativecommons.org/licenses/by-nc/4.0/). 